\title{
Les espaces libres et leur préservation dans les régions urbaines du Rhin supérieur méridional : Bâle/Fribourg-en-Brisgau/Mulhouse
}

Open space preservation and land use for recreational purposes in metropolitan areas of the Southern Rhine Valley: a comparison of the Basle, Friburg and Mulhouse urban areas

Freiraumsicherung und Freiräume in den Stadtregionen des Südlichen Oberrheins : Basel, Freiburg i. Br. und Mulhouse im Vergleich

\section{Martin Sandtner}

\section{OpenEdition}

\section{Journals}

\section{Édition électronique}

URL : http://journals.openedition.org/rge/2794

DOI : 10.4000/rge.2794

ISSN : 2108-6478

Éditeur

Association des géographes de l'Est

Édition imprimée

Date de publication : 1 janvier 2002

ISSN : 0035-3213

Référence électronique

Martin Sandtner, «Les espaces libres et leur préservation dans les régions urbaines du Rhin supérieur méridional : Bâle/Fribourg-en-Brisgau/Mulhouse », Revue Géographique de l'Est [En ligne], vol. 42 / 1-2 I 2002, mis en ligne le 05 janvier 2011, consulté le 08 septembre 2020. URL : http:// journals.openedition.org/rge/2794; DOI : https://doi.org/10.4000/rge.2794

Ce document a été généré automatiquement le 8 septembre 2020

Tous droits réservés 


\section{Les espaces libres et leur} préservation dans les régions urbaines du Rhin supérieur méridional : Bâle/Fribourg-enBrisgau/Mulhouse

Open space preservation and land use for recreational purposes in metropolitan areas of the Southern Rhine Valley: a comparison of the Basle, Friburg and Mulhouse urban areas

Freiraumsicherung und Freiräume in den Stadtregionen des Südlichen Oberrheins : Basel, Freiburg i. Br. und Mulhouse im Vergleich

Martin Sandtner

\section{Introduction : la notion de « ville intermédiaire » existe- t-elle dans l'espace du Rhin supérieur?}

L'extension spatiale résidentielle a été l'une des caractéristiques principales de la transformation du paysage d'Europe centrale au cours des dernières décennies: de vieux noyaux de peuplement se sont élargis de manière concentrique en de nouvelles aires résidentielles; aux alentours des grandes villes, des villages agricoles se sont transformés en communes-dortoirs de migrants quotidiens; même des communes situées à une certaine distance des centres urbains connaissent depuis quelques années un réel «boom » immobilier, un développement propre aux aires métropolitaines en cours de constitution. Les grands espaces libres continus se sont raréfiés entre-temps jusqu'à une importante distance des concentrations urbaines; les superficies construites ont fusionné, formant des conurbations qui constituent aujourd'hui un 
tissu à grande échelle d'aires résidentielles et professionnelles, reliées entre elles par des axes de communication fortement sollicités.

Pour l'appellation de cette nouvelle réalité urbaine, Sieverts (1999) a eu recours au terme très expressif de "Zwischenstadt », ville intermédiaire, « la ville entre les villes » (the city between cities). Il ne s'agit pas ici, bien entendu, de la notion utilisée par les géographes pour désigner un échelon intermédiaire entre la métropole et le centre régional, mais du paysage urbain qui se développe entre deux grandes villes. Selon Sieverts, cette ville intermédiaire est très souvent un espace sans visage, qui n'est ni ville ni milieu rural, ni centre ni périphérie. Il relève notamment la présence de lotissements, de maisons monofamiliales qui ont trouvé refuge dans les communes suburbaines, de grands ensembles d'habitation uniformes ou presque, ainsi que de zones d'activité géantes. Il qualifie très négativement ces phénomènes de "bouillies de peuplement», de "paysages habités déstructurés», de "paysages consommés ", voire de "déserts de peuplement ", phénomènes signalés aussi par de nombreuses publications parues durant les années 1970-1980 sur la «contreurbanisation ».

3 Pour Sieverts, la ville intermédiaire est une réalité nouvelle et non la reproduction dépassée de l'évolution de la vieille ville européenne dense. Cet auteur milite en faveur d'une vitalisation et d'une spécialisation de la ville intermédiaire, à laquelle il convient de conférer une identité, dont il faut développer les potentialités. Il importe de rendre «lisibles » les «non-lieux» (Marc Augé) et, par là-même, de montrer que la ville intermédiaire est digne d'être vécue. A cet effet, les espaces libres, disponibles, sont déterminants pour une ville intermédiaire ; ils doivent servir en fait de charnière. C'est à partir d'eux que la ville intermédiaire mérite d'être vécue. Les espaces libres sont susceptibles de maintenir ou de forger une identité, de créer du lien ou du liant.

La région du Rhin supérieur méridional révèle des indices qui dénotent une certaine évolution vers la ville intermédiaire. Les villages urbanisés et les effets inadaptés des grands ensembles résidentiels ne sont pas rares dans chacune des trois portions nationales de la Regio TriRhena. Il convient de se demander dès à présent quel est le degré de progression de cette évolution vers la ville intermédiaire dans les régions urbaines de Bâle, Fribourg-en-Brisgau et Mulhouse. Ne trouve-t-on dans l'espace périphérique éloigné de ces centres que des aires résidentielles sans visage pour les travailleurs des villes ? Un discours qui se retrouve dans la stratégie à l'américaine de la recentralisation, de la polarisation, du «mixage» des hommes et des fonctions. La Regio TriRhena serait-elle même en train de se développer en une seule ville intermédiaire transfrontalière ? Où le contraste entre la ville et la campagne continuet-il à se manifester par des espaces urbains nettement délimités, entourés de larges espaces libres ? Existe-t-il par ailleurs des différences entre les trois régions urbaines? De quelle façon les aires de peuplement, les aires construites et celles encore libres de toute affectation sont-elles agencées? Les espaces libres encore disponibles de la ville intermédiaire, s'il y en a, sont-ils à même de lui conférer un visage, de rendre cette ville viable, durable?

5 Le présent article vise à répondre à ces questions par une analyse qui s'appuie sur le "Système d'information géographique " (SIG). Le périmètre de l'investigation est constitué par les trois villes et l'espace environnant du Rhin supérieur méridional (cf. fig. 1 de la contribution Eder-Sandtner \& Sandtner, en ce volume). Les trois villes sont très comparables au point de vue de leur nombre d'habitants (Bâle : 166000 habitants ; 
Fribourg-en-Brisgau: 200000 habitants; Mulhouse : 110000 habitants). Le premier paragraphe examine la pertinence des fonctions centrales que les espaces libres sont à même d'assumer aujourd'hui en faveur de la population des agglomérations urbaines. Le deuxième paragraphe décrit la base de données et la méthode. Enfin, le troisième présente et interprète les résultats obtenus pour les trois villes.

\section{Les espaces libres proches des aires de peuplement : l'espace de récréation devant la porte de la maison d'habitation}

6 Dans les agglomérations, les espaces libres, sans être forcément publics, sont recherchés et utilisés pour des raisons récréatives; le loisir les identifie à l'espace (libre). A travers la fonction récréative, les espaces libres façonnent ainsi l'image que retiennent les habitants de leur environnement résidentiel immédiat. Cette étude est consacrée à la qualité spatiale de la ville intermédiaire, qui est influencée de manière déterminante par la possibilité de pratiques récréatives de proximité. Aussi ne comporte-t-elle pas l'analyse d'autres fonctions, quoiqu'importantes, relatives à la production d'air pur, aux réserves en eau ou aux réserves foncières destinées au développement du transport et à celui du logement.

7 Selon Müller, Kramer \& Ferrante (1997), le temps récréatif inhérent au loisir est à percevoir comme un ensemble de «séquences chronologiques à forte autonomie temporelle ». Le loisir est quotidien, hebdomadaire et annuel ; les séquences récréatives relativement courtes l'emportent nettement: en Allemagne les enquêtes («Deutsche Gesellschaft für Freizeit » 1999) ont établi que $38 \%$ du temps de loisir sont consacrés aux loisirs quotidiens, c'est-à-dire vécus durant les jours ouvrables, surtout en fin de journée, et seulement $21 \%$ aux congés. Comme il reste ainsi peu de temps pour les activités de loisir à grande distance, les besoins en aires de loisir de proximité sont amples, une proximité qui peut être pensée à partir des lieux de scolarisation, de travail... Ces besoins sont renforcés par les nombreuses catégories démographiques à mobilité réduite, telles que celles des enfants et des personnes qui en ont la charge, des adolescents et des personnes âgées réduites à consommer leur temps libre disponible dans un rayon compatible avec la marche à pied.

Dès lors que l'on examine les activités récréatives les plus courantes, on observe la prédominance d'activités plutôt passives passées au domicile: la consommation médiatique apparaît avec un certain recul comme l'activité récréative la plus fréquente (Müller, Kramer \& Ferrante, 1997). Les notions de "nature », " santé » et «paysage» sont en outre considérées comme les plus importantes pour les loisirs («Deutsche Gesellschaft für Freizeit», 1999). Parmi les activités récréatives horsdomicile les plus appréciées figurent la promenade et le cyclisme (Opaschowski, 1997) ; selon une enquête expressive à cet égard conduite en Suisse alémanique, au moins $48 \%$ des interviewés s'adonnent à la promenade pédestre hebdomadaire (Meyrat-Schlee, 1992). Cette promenade qui part du domicile figure toujours encore parmi les activités récréatives déterminantes.

9 Dans ce cadre, les exigences spatiales sont minimes (Kiemstedt \& Scharpf 1990, Jessel 1998, Kaplan 1988) et les investissements infrastructurels nuls, contrairement à la pratique sportive diversifiée, dans la mesure où les intéressés peuvent avoir recours au 
réseau routier et aux chemins existants. Les promeneurs sont particulièrement intéressés par des paysages offrant des divertissements variés. Ils souhaitent un balisage adéquat des itinéraires favorisant la mise en valeur des lisières forestières, des ruisseaux et rivières ainsi que d'immeubles marquants. Une spécificité naturelle ou culturelle, c'est-à-dire la présence d'éléments paysagers locaux typiques dans une constellation expressive accentue en outre l'attractivité d'un espace. Une promenade est en fin de compte susceptible de satisfaire à l'aspiration à la nature, étant bien entendu que la notion de nature n'est pas à confondre avec une nature physique non anthropisée, au sens écologique pur du terme. Ici la nature est conçue comme une construction esthétique, même si ce n'est qu'une allée verte ou un parc paysager entretenu. Le trafic routier est particulièrement perçu comme une nuisance : le bruit dérange cette détente tranquille justement recherchée pendant la promenade.

La disponibilité d'espaces correspondant à ces exigences est aujourd'hui surtout fortement réduite, quantitativement et qualitativement, par l'extension des aires de peuplement et l'accroissement du trafic individuel motorisé. Les espaces libres à grande extension, sur lesquels la nature peut être vécue au sens évoqué ci-dessus, font la plupart du temps défaut dans les régions urbaines. Même le paysage urbain quotidien, c'est-à-dire les rues, chemins et places, est de moins en moins approprié à la promenade; les parcs de verdure sont eux aussi très rarement libres de nuisances inhérentes à la circulation motorisée (Busch, Preuck \& Reckhard, 1983).

11 L'accent est mis ainsi sur le manque essentiel inhérent à la ville intermédiaire, à savoir l'absence d'espaces à haute qualité attractive susceptibles de favoriser une vie récréative résidentielle de proximité et de promouvoir par là-même l'identification des habitants avec l'environnement dans lequel ils vivent quotidiennement, quoique l'urbanité passe aussi par la fréquentation d'espaces "pleins», "occupés», fonctionnalisés.

\section{Données de base et méthodologie}

12 Les nécessités d'une étude comparée de l'état des espaces libres dans les trois agglomérations de Bâle, Fribourg-en-Brisgau et Mulhouse impliquent une démarche fondée sur une base de données commune et une méthodologie unifiée. La présente subdivision vise à mettre l'accent sur les données relatives à un niveau d'abstraction spatiale sommaire, qui sont disponibles pour l'ensemble de l'Union européenne et la Suisse; elles permettent ainsi des comparaisons à l'échelle européenne. Siedentop (1999) a mis au point une méthode qui permet de comparer sur cette base les régions urbaines entre elles. Cette méthode a également été appliquée au cas du Rhin supérieur méridional.

\section{Le projet « Land cover data » CORINE}

13 Le projet "Land cover data" CORINE ("Co-ordination of information on the environment») de l'Union européenne a permis pour la première fois durant les années 1990 de réunir à l'échelle de la totalité de cette Union des données statistiques comparables sur l'usage du sol, autorisant une cartographie transnationale. Celle-ci est fondée sur une interprétation visuelle d'images satellitaires, complétée par le recours à des photographies aériennes et à des cartes topographiques. Une clé d'interprétation à 
trois niveaux a donné lieu à l'élaboration de 44 formes de couverture du sol à l'échelle 1/100 000. Au niveau médian (niveau 2) ces formes sont réduites à 15 types. Les images satellitales (Landsat-TM) qui constituent la base de données de CORINE ont l'inconvénient de remonter aux années 1990 et 1991, à la fois pour le Bade-Sud et le Haut-Rhin ; elles ne peuvent donc plus être considérées comme actuelles, compte tenu de la rapidité du processus d'urbanisation. Parallèlement à cette insuffisante actualisation, Prechtel (1997) met l'accent sur un autre point critique : celui du degré très prononcé de généralisation sur lequel reposent ces données. Le seuil inférieur de l'enquête s'est situé autour de 25 ha pour l'observation des superficies; les objets linéaires n'ont été cartographiés qu'à partir d'une largeur de 100 mètres. Ce procédé sous-évalue systématiquement les couvertures à surface réduite.

Pour combler la lacune existant sur la carte européenne en ce qui concerne la Suisse, Etat non communautaire, les données statistiques helvétiques, appuyées sur l'interprétation de photographies aériennes, ont été adaptées à la classification CORINE (cf. Office fédéral de la statistique \& l'office fédéral pour l'environnement, les forêts et paysages, 1998). Pour la Suisse du Nord-Ouest, les photographies aériennes qui ont servi de base de données remontent à 1982 ; elles sont donc encore moins actuelles que les bases utilisées pour le Bade-Sud et l'Alsace.

Pour faire ressortir les caractéristiques de l'état des espaces libres dans les régions urbaines du Rhin supérieur méridional, les données relatives aux composantes badoise, alsacienne et suisse ont été réunies en un système commun de coordination. Cette démarche a permis d'avoir un aperçu sur l'usage des sols et par là-même sur les espaces libres disponibles ainsi que sur leur répartition. En dépit des insuffisances signalées, la base de données CORINE et son complément statistique suisse constituent une première source d'informations comparables, à l'aide desquelles la région frontalière peut être considérée dans une dimension proprement régionale.

\section{L'analyse SIG du modèle d'aires libres et d'aires de peuplement}

Pour pouvoir comparer plusieurs régions urbaines entre elles, outre la base de données, la définition de la délimitation spatiale est déterminante. Il serait possible de recourir à cet effet à la délimitation administrative. Mais ce procédé est à écarter dans la mesure où les frontières ont été façonnées en fonction de circonstances historiques et de hasards. Il n'est pas possible non plus d'appliquer aux régions frontalières des critères socio-démographiques ou structurels à la délimitation des agglomérations, aucune méthode uniforme internationale de délimitation des périmètres d'agglomération n'ayant été mise au point. C'est pourquoi il convient de privilégier la présente démarche fondée sur des délimitations géométriques. Les travaux de Siedentop (1999) s'appuient sur un rayon défini de 20 kilomètres autour du centre du noyau urbain. Autour de la ville centrale, l'espace est subdivisé à intervalles réguliers d'un kilomètre par des cercles concentriques. Ce procédé permet de calculer et de représenter au moyen du SIG des grandeurs structurelles en état de dépendance de la distance par rapport au centre. La ville et son espace ambiant sont en outre subdivisés en huit secteurs géométriques identiques représentés par des cercles. Il est ainsi possible de reconnaître les asymétries dans l'évolution de l'occupation du sol. La figure 1 illustre ce système de rapport spatial à partir de la région bâloise. 
Figure 1 : Système de rapport spatial, selon l'analyse SIG, illustré par le cas de la région bâloise

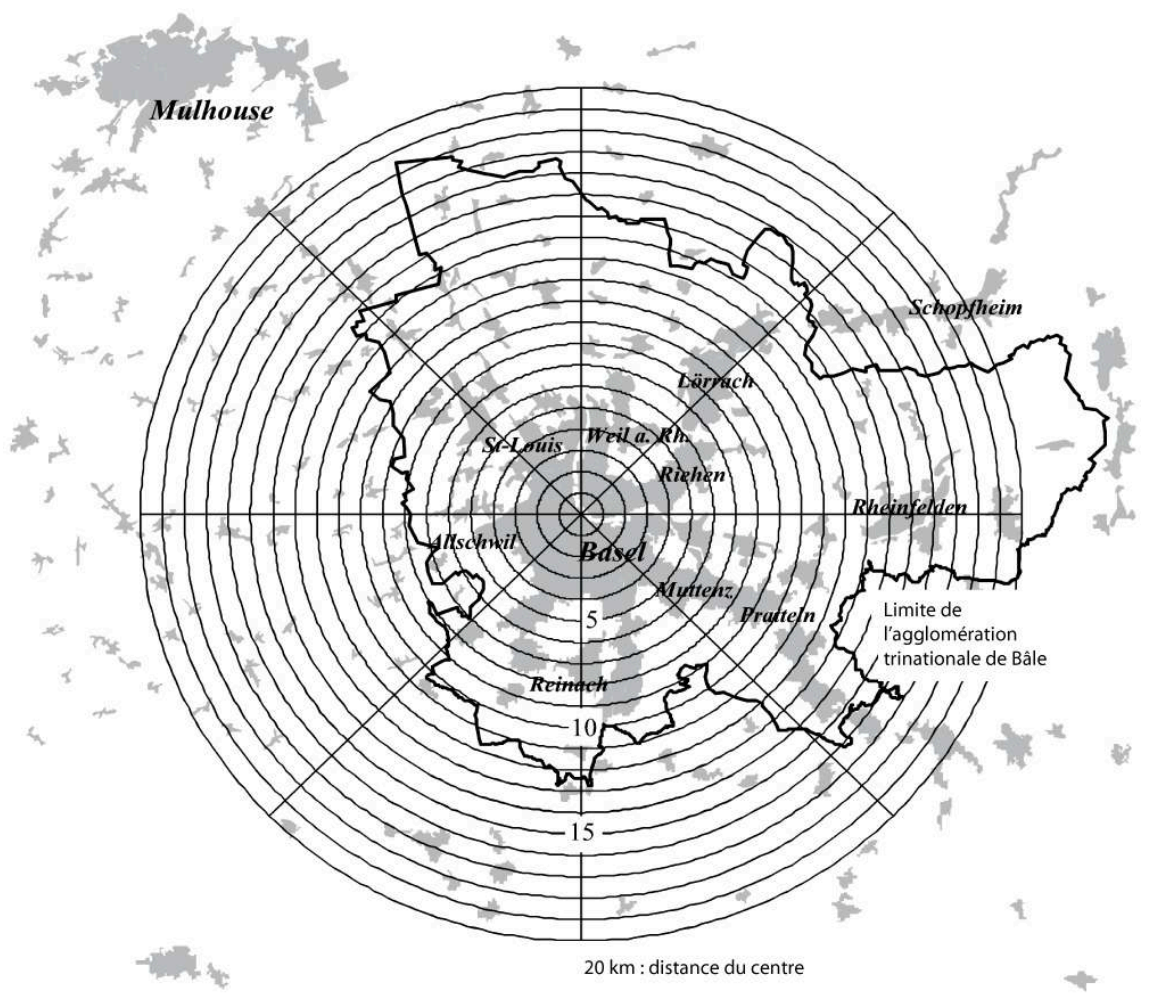

17 A l'aide des données de base décrites ci-dessus et du système de rapport spatial, il est possible de calculer de nombreuses valeurs de référence qui permettent de cerner l'état des espaces libres et de leur disponibilité. Le tableau 1 en énumère et décrit les indicateurs. La comparaison de plusieurs régions urbaines entre elles permet une relative qualification de l'état des espaces libres. 
Tableau 1 : Valeurs de référence pour une description comparée de l'état des espaces libres à l'échelle régionale

\begin{tabular}{|c|c|c|}
\hline Valeur de référence & Nature du rapport spatial & Fonction ou aptitude à la fonction récréative \\
\hline$\%$ d'espaces libres & $\begin{array}{l}\text { \% d'espaces libres par rapport } \\
\text { à la surface totale }\end{array}$ & $\begin{array}{l}\text { l'accomplissement de l'aspiration au vécu dans } \\
\text { les paysages ouverts n'est possible que sur des } \\
\text { espaces libres d'une certaine ampleur }\end{array}$ \\
\hline $\begin{array}{l}\text { gradient ville-espace } \\
\text { périphérique }\end{array}$ & $\begin{array}{l}\text { \% d'occupation du sol/km } \\
\text { d'aire circulaire autour du } \\
\text { centre-ville }\end{array}$ & $\begin{array}{l}\text { cet indicateur est une mesure du degré } \\
\text { d'urbanisation de la périphérie. Si l'espace } \\
\text { construit dense est limité à quelques kilomètres } \\
\text { autour du centre-ville, il s'agit d'une région } \\
\text { urbaine compacte, qui offre aux habitants, à faible } \\
\text { distance, des possibilités conjuguées d'espaces libres } \\
\text { epanonouissants. Lorsque le gradient demeure faible } \\
\text { jusqu'à une grande distance du centre-ville, le haut } \\
\text { degré d'urbanisation atteint entraîne une faible dis- } \\
\text { ponibilité d'espaces libres }\end{array}$ \\
\hline $\begin{array}{l}\text { rapport périphérie- } \\
\text { contenu du système } \\
\text { d'occupation du sol }\end{array}$ & $\begin{array}{l}\text { rapport longueur du périmètre } \\
\text { de peuplement/racine de } \\
\text { l'aire de peuplement }\end{array}$ & $\begin{array}{l}\text { «mesure de compacité » du système d'occupation } \\
\text { du sol ; à la périphérie de l'espace construit } \\
\text { la dégradation de l'image paysageree est } \\
\text { particulièrement forte ; ici une aire d'occupation } \\
\text { désordonnée est à évaluer négativement sous l'angle } \\
\text { esthétique. Par contre une grande et longue aire de } \\
\text { peuplement périnhérique offre, } \\
\text { au regard de son contenu, une forte alternance d'es- } \\
\text { paces occupés et d'espaces libres, et par là-même, } \\
\text { une bonne accessibilité de ces derniers }\end{array}$ \\
\hline $\begin{array}{l}\text { desserte en espaces } \\
\text { libres des aires à } \\
\text { empreinte urbaine }\end{array}$ & $\begin{array}{l}\text { \% des aires à empreinte } \\
\text { urbaine localisées à } 500- \\
1000 \mathrm{~m} \text { de l'espace libre } \\
\text { le plus proche/surface totale }\end{array}$ & $\begin{array}{l}\text { espaces occupés sans espaces libres (suffisamment } \\
\text { grands) en nombre satisfaisant. La distance de } \\
500 \text { à } 1000 \text { m correspond à un chemin piétonnier } \\
\text { de } 7 \text { à } 15 \text { minutes environ. Il est ainsi possible } \\
\text { à empreinte urbaine d'identifier des espaces occupés } \\
\text { dans lesquels les espaces verts et/ou les voies de cir- } \\
\text { culation intraurbains favorisent une qualité résiden- } \\
\text { tielle élevée. La part de ces espaces dans la superficie } \\
\text { totale du sol occupé est un indicateur pour la poll- } \\
\text { tique en mateiere d'espaces libres en général }\end{array}$ \\
\hline$\%$ de forêts & $\begin{array}{l}\% \text { de forêts par rapport } \\
\text { à la superficie totale }\end{array}$ & $\begin{array}{l}\text { les aires forestières sont particulièrement } \\
\text { importantes pour la détente tranquille en pleine } \\
\text { nature. La part de la forêt est ainsi un indicateur pour } \\
\text { la qualité récréative de la région urbaine dans son } \\
\text { ensemble }\end{array}$ \\
\hline $\begin{array}{l}\text { aires forestières } \\
\text { à proximité des aires } \\
\text { d'occupation du sol } \\
\text { de peuplement }\end{array}$ & $\begin{array}{l}\text { \% d'aires forestières } \\
\text { par rapport aux espaces } \\
\text { libres proches des aires } \\
\text { (distance }<\text { ou }=500 \mathrm{~m} \\
\text { des aires à empreinte } \\
\text { urbaine) }\end{array}$ & $\begin{array}{l}\text { cet indicateur est expressif du degré d'accessibilité } \\
\text { aux aires forestières depuis les aires de } \\
\text { peuplement. II permet de concrétiser } \\
\text { le degré de participation de la forêt à a fonction } \\
\text { récréative de l'ensemble du périmètre étudié }\end{array}$ \\
\hline
\end{tabular}

D’après Siedentop, 1998, modifié et complété

\section{La situation comparée des espaces libres dans les régions de Bâle, Fribourg-en-Brisgau et Mulhouse}

\section{Aires d'occupation du sol et espaces libres}

La figure 2 donne un aperçu de l'état des aires d'occupation du sol et de celles des espaces libres dans les trois régions urbaines. Est représenté le niveau 2 des données CORINE, sous la forme des quatre classes d'usage du sol déterminantes pour l'analyse de l'état des espaces libres, à savoir les " aires à empreinte urbaine ", « les aires d'activités $\mathrm{du}$ secteur secondaire, les aires affectées au transport et à l'exploitation du sol et du sous-sol, aux dépôts d'ordures et à la construction", «la forêt » et «les aires libres diverses ». Les indicateurs présentés à propos de l'étude sur l'état des espaces libres figurent sur le tableau récapitulatif 2. 
Figure 2 : Comparaison de l'usage du sol dans les régions urbaines de Bâle, Fribourg-en-Brisgau et Mulhouse, selon la base de données CORINE

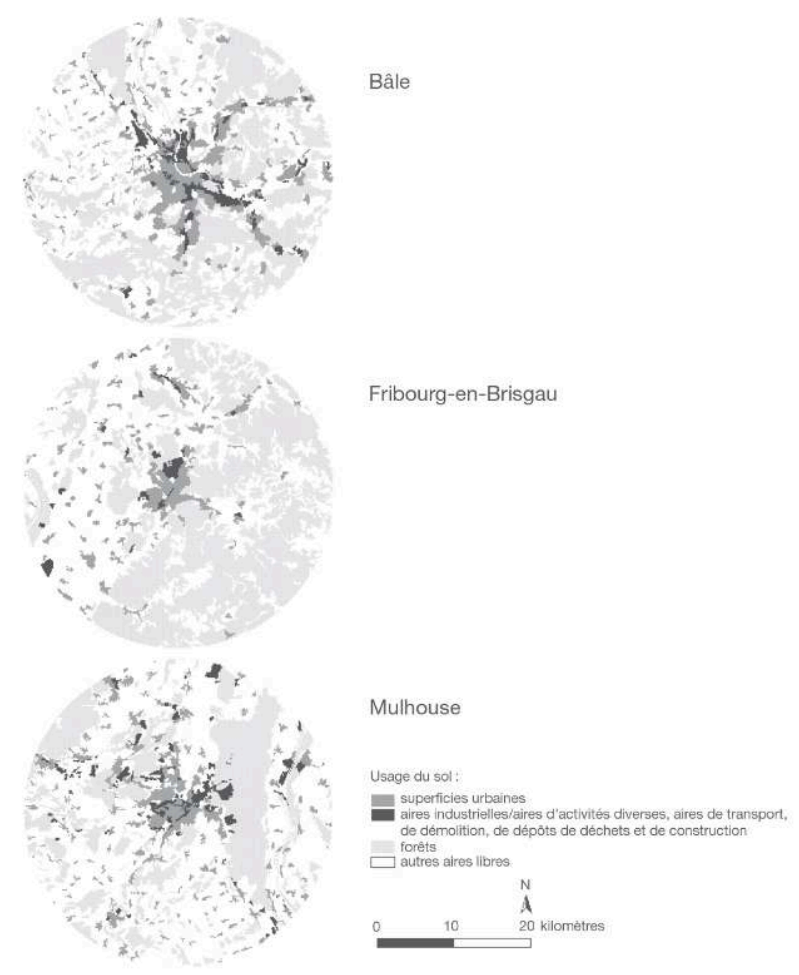

Sources : pour la Suisse, Office fédéral de la statistique ; pour l'Allemagne, Office fédéral de la statistique ; pour la France, Institut français de l'environnement. Années de référence : 1982 pour la Suisse, 1990 pour l'Allemagne, 1991 pour la France

\section{Parts de peuplement et espaces libres}

La figure 3 montre l'importance de la part de la superficie de peuplement dans les huit périmètres d'un rayon de $20 \mathrm{~km}$ chacun autour des centres-villes de Bâle, Fribourg-enBrisgau et Mulhouse. Chaque région urbaine est représentée par une ligne. En ce qui concerne le secteur ESE, la figure exprime par exemple que dans la région bâloise $23 \%$, dans la région mulhousienne à peine $10 \%$ et dans la région fribourgeoise $4 \%$ de la superficie totale sont des surfaces de peuplement. 
Figure 3 : Part de l'aire de peuplement par rapport à la superficie totale, en \%, par secteur dans les régions urbaines de Bâle, Fribourg-en-Brisgau et Mulhouse

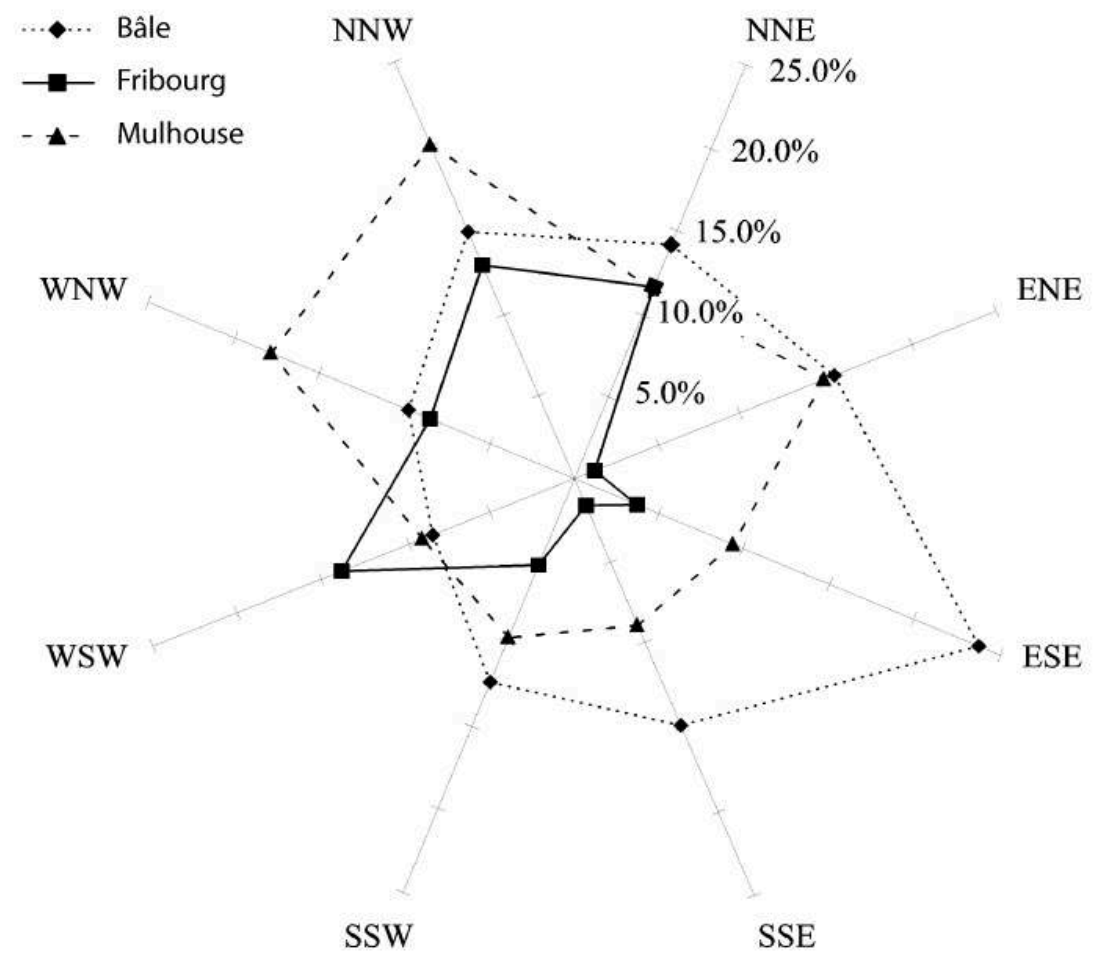

Sources et années de référence : cf. fig. 2 autour des noyaux urbains s'est effectuée de façon symétrique ou, le cas échéant, asymétrique, par suite de contraintes naturelles ou culturelles. La prise en compte de cette donnée est importante pour l'accessibilité d'espaces libres à partir du centre-ville et en général pour la qualité de la vie et l'attractivité d'une ville. La région de Fribourg, en raison de son relief, est par exemple densément peuplée dans les secteurs occidentaux, alors que dans la région bâloise les secteurs occidentaux sont les plus faiblement peuplés par suite de la proximité de la frontière internationale. Bâle et son espace environnant occupent l'essentiel de l'espace considéré. Cela signifie qu'inversement la part des aires libres dans la superficie totale est ici la moins élevée, avec $85,9 \%$, contre $87,4 \%$ pour Mulhouse et $92,7 \%$ pour Fribourg (tableau 2). Les surfaces construites s'étendent de façon tentaculaire depuis le noyau urbain de Bâle jusqu'aux vallées proches. Ces aires de peuplement se déroulent en grande partie en bandes continues. La structure immobilière très ramifiée s'étend loin dans l'espace environnant. Sous sa forme compacte, elle s'exprime cependant aussi dans un rapport moyen périmètre/contenu du système de peuplement de 67,2 (tableau 2). 
Tableau 2: Valeurs de référence pour une description comparée de l'état des espaces libres à l'échelle régionale dans les trois régions urbaines de Bâle, Fribourg-en-Brisgau et Mulhouse.

\begin{tabular}{|c|c|c|c|c|}
\hline Indicateur & $\begin{array}{l}\text { Nature du rapport spatial } \\
\text { (selon Siedentop 1998) }\end{array}$ & $\begin{array}{l}\text { Bâle } \\
\text { en } \%\end{array}$ & $\begin{array}{l}\text { Fribourg } \\
\text { en } \%\end{array}$ & $\begin{array}{l}\text { Mulhouse } \\
\text { en } \%\end{array}$ \\
\hline $\begin{array}{l}\% \text { du total } \\
\text { des aires libres }\end{array}$ & $\begin{array}{l}\% \text { des aires libres par } \\
\text { rapport à la surface totale }\end{array}$ & 85,9 & 92,7 & 87,4 \\
\hline $\begin{array}{l}\text { rapport périmètre/ } \\
\text { contenu du système } \\
\text { de peuplement }\end{array}$ & $\begin{array}{l}\text { longueur du périmètre } \\
\text { du peuplement/racine de } \\
\text { l'aire de peuplement }\end{array}$ & 67,2 & 52,4 & 85,4 \\
\hline $\begin{array}{l}\text { les aires d'empreinte } \\
\text { urbaine et leurs espaces } \\
\text { libres }\end{array}$ & $\begin{array}{l}\text { part des aires d'empreinte } \\
\text { urbaine distantes de l'espace } \\
\text { libre le plus proche, de: } \\
\text { - plus de } 500 \mathrm{~m} \\
\text { - plus de } 1000 \mathrm{~m} \\
\text { par rapport à la surface totale } \\
\text { d'empreinte urbaine }\end{array}$ & $\begin{array}{l}9,7 \\
1,1\end{array}$ & $\begin{array}{l}7,4 \\
0,4\end{array}$ & $\begin{array}{r}12,7 \\
7,5\end{array}$ \\
\hline part de la forêt & $\begin{array}{l}\text { part des aires forestières } \\
\text { par rapport à la surface totale }\end{array}$ & 37,5 & 45,7 & 29,9 \\
\hline $\begin{array}{l}\text { aires forestières à } \\
\text { proximité de l'espace } \\
\text { de peuplement }\end{array}$ & $\begin{array}{l}\text { part des aires forestières } \\
\text { par rapport aux espaces } \\
\text { libres proches des aires } \\
\text { de peuplement (distance } \\
<\text { ou }=500 \mathrm{~m} \text { des aires à } \\
\text { empreinte urbaine) }\end{array}$ & 24,0 & 18,1 & 14,8 \\
\hline
\end{tabular}

Sources et années de référence : cf. figure 2

Mulhouse, alors qu'il a été possible de concentrer essentiellement, au moins jusqu'à présent, l'occupation du sol en direction des axes constitués par les vallées. Celles-ci n'ont toutefois guère davantage d'aires libres importantes d'un seul tenant susceptibles de servir de ceinture d'interruption du peuplement à valeur récréative élevée. Fribourg par contre ne s'est étendue jusqu'à présent que très peu en direction de l'espace voisin. Les communes de la périphérie n'ont pas fusionné spatialement en une «bouillie de peuplement » indifférenciée ; elles demeurent séparées entre elles par de grandes aires libres.

\section{Gradient ville/campagne}

Le gradient ville-campagne de Fribourg, dont l'aire de peuplement atteint $85,5 \%$ en centre-ville, diminue très vite ensuite; déjà dans la zone du cercle concentrique 5 (éloignée de 4 à $5 \mathrm{~km}$ du centre-ville), il tombe en-dessous de $20 \%$; toutes les zones à partir de la zone 6 sont sous les $6 \%$. A Bâle la zone 1 atteint $86,7 \%$ - le Rhin constitue ici une aire libre -, tandis que la zone 2 monte à $93,6 \%$. Mais la zone 5 n'accuse plus qu'à peine $50 \%$. Le taux diminue régulièrement en direction de la sortie de la ville. Ce n'est qu'en zone 10 que le taux tombe à moins de $20 \%$; il n'est plus que de $10 \%$ dans la zone 13. Observé à ce niveau d'échelle, le centre-ville de Mulhouse est complètement construit; mais au fur et à mesure que l'on s'en éloigne, le taux d'occupation du sol 
diminue très vite. La zone 5 n'est plus construite qu'à raison de $34,7 \%$. En zone 8 le taux est inférieur à $20 \%$ et en zone 10 il est inférieur à $10 \%$. Il convient toutefois de relever que les zones formées par les cercles périphériques accusent les taux les plus élevés : ici le Haut-Rhin compte de nombreux villages qui ont connu récemment une croissance soutenue dans le cadre de la sub- et de la contre-urbanisation.

Figure 4 : Gradient ville-campagne comparé des trois régions urbaines de Bâle, Fribourg et Mulhouse

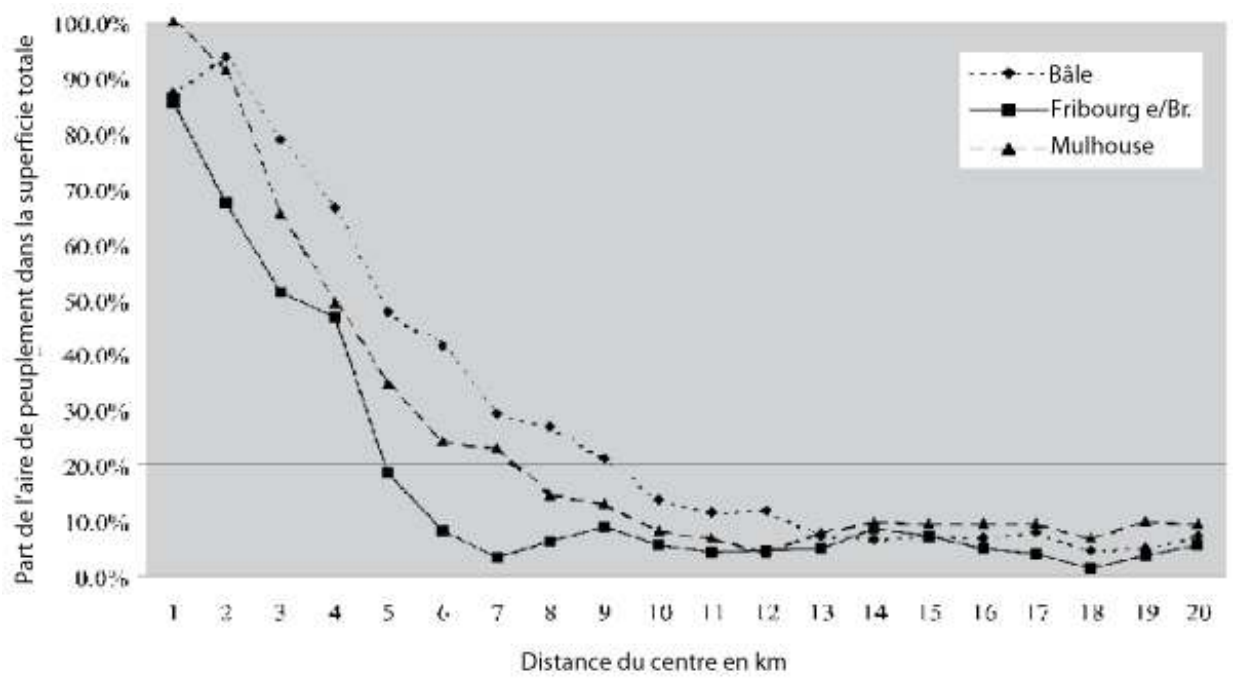

Sources et années de référence : cf. fig. 2

\section{Répartition sectorielle de parts d'aires d'occupation du sol et d'espace libre}

La région urbaine de Fribourg se caractérise nettement par son asymétrie (fig. 4). Alors que les territoires situés à l'est du centre n'ont que des taux d'occupation du sol de 1,1 à $3,7 \%$, les secteurs ouest-sud-ouest, nord-nord-ouest et nord-nord-est ont une densité relativement élevée, avec des taux respectifs de 13,8,12,9 et 11,6\%. Ici s'affirme la limite naturelle entre la plaine du Rhin supérieur et l'espace collinaire adjacent d'une part, la Forêt-Noire d'autre part. Pendant qu'en montagne moyenne seules quelques petites aires de peuplement sont à dénombrer dans les vallées, le paysage collinaire fertile comporte de nombreuses localités d'origine ancienne.

La région urbaine de Bâle ne s'est pas développée non plus de manière uniforme, d'autant plus que la frontière a joué dans ce mouvement la part essentielle : le secteur est-sud-est, traversé par l'«Ergolztal» situé dans le canton de Bâle-campagne, enregistre le taux le plus élevé en matière d'aire de peuplement, soit $23,7 \%$. Les autres secteurs qui se trouvent entièrement ou pour partie sur le territoire suisse, sont également densément peuplés. Les secteurs localisés dans le nord, comportant l'aéroport et les communes françaises environnantes, ainsi que le "Wiesental» allemand ont toutefois un taux d'environ $15 \%$ d'espace de peuplement. Les secteurs ouest-sud-ouest et ouest-nord-ouest sont bien moins peuplés (respectivement 8,3 et $9,7 \%$ ). Cette aire se caractérise, notamment en territoire français, par des localités plus petites demeurées en grande partie à l'écart de la suburbanisation. 
27 Les différences sectorielles en matière d'occupation du sol sont moins nettes dans la région de Mulhouse. Il apparaît nettement que les quatre secteurs méridionaux, situés sous la barre de $10 \%$, sont moins peuplés que les quatre secteurs septentrionaux qui ont des taux évoluant entre tout juste 10 et $20 \%$. Dans le nord de la ville se trouvent d'une part d'importants ensembles d'habitation suburbains et quelques zones industrielles, d'autre part aussi des aires très étendues de mines de potasse en voie de reconversion.

\section{Aires d'empreinte urbaine et espaces libres}

28 Cet indicateur (tableau 2) permet l'évaluation de l'accessibilité aux espaces libres à partir des aires d'habitation. La situation est particulièrement expressive en ce qui concerne la population de la région fribourgeoise: seulement $7,4 \%$ des aires à empreinte urbaine sont situées à plus de $500 \mathrm{~m}$ à vol d'oiseau de l'espace libre le plus proche. $0,4 \%$ seulement des aires habitées sont à plus de $1000 \mathrm{~m}$ d'un espace libre. Cette région urbaine, dont le bâti continu de l'agglomération centrale est relativement réduit, et qui n'a à faire face qu'à de rares zones industrielles et d'activités en général, est ainsi bien pourvue en espaces libres de proximité.

La forte ramification structurelle des aires à empreinte urbaine de la région bâloise explique la raison pour laquelle les espaces libres sont également ici très peu distants des aires d'habitation : seuls 9,7 \% des aires à empreinte urbaine sont à plus de $500 \mathrm{~m}$ de l'espace libre le plus proche ; à peine $1,1 \%$ d'entre elles en sont éloignées de plus de $1000 \mathrm{~m}$. Ces aires sont en grande partie localisées dans la ville de Bâle même, qui ne possède que peu d'espaces libres de plus de 25 ha (seuil inférieur de l'enquête cartographique menée selon le procédé CORINE), et qui jouxtent surtout au nord ainsi qu'au sud-est de grandes aires industrielle et manufacturières, de même que des aires réservées au transport. Les communes agglomérées localisées sur les axes des vallées sont par contre bien dotées en espaces libres, selon la méthode d'investigation utilisée. Les espaces libres de taille réduite et les aires construites ont surtout une grande importance à l'intérieur de la ville : aménagés en conséquence, ils se prêtent très bien aux promenades. La base de données utilisée ne permet cependant pas d'analyse fine de cet aspect en ce qui concerne la composante qualitative des espaces libres, à savoir l'intensité des usages, de la structure en général, du relief et de bien d'autres critères.

30 En matière d'espaces libres disponibles, la situation apparaît toutefois plus critique à Mulhouse. Dans cette région urbaine, 12,7 et 7,5\% des aires à empreinte urbaine sont éloignées respectivement de plus de $500 \mathrm{~m}$ et de plus de $1000 \mathrm{~m}$ de l'espace libre le plus proche. Ici les aires d'habitation avoisinent fréquemment les zones industrielles et d'activités artisanales qui ne sont plus ou que très peu appropriées à l'aménagement d'aires de détente. L'aire construite en continu du noyau urbain et des communes environnantes a une taille comparable à celle de Bâle; à Mulhouse cependant la " coulée verte ", c'est-à-dire des aires libres continues aux profondes avancées dans la zone de peuplement, sources de déstructuration, existent à peine. Cette situation explique le relatif déficit en espaces libres. 


\section{Rapports de proximité entre les aires forestières et celles à empreinte urbaine}

Presque la moitié de l'espace au-delà d'un rayon de $20 \mathrm{~km}$ autour de Fribourg est boisé (tableau 2 et fig. 2). La Forêt-Noire toute proche offre en altitude de vastes aires forestières continues à valeur récréative élevée. La plaine rhénane qui borde la ville à l'ouest et au nord, ainsi que le «Kaiserstuhl» sont également couverts d'aires forestières. Celles-ci sont toutefois rares à proximité immédiate des aires à empreinte urbaine ( $18,1 \%$ des espaces libres sont boisés jusqu'à $500 \mathrm{~m}$ de distance); ici les aires à dominante agricole l'emportent.

Dans la région bâloise 37,5 \% de la surface totale sont couverts par la forêt. Les grandes aires forestières sont surtout localisées sur le plateau et dans les chaînes jurassiennes, ainsi que sur le «Dinkelberg » et les promontoires méridionaux de la Forêt-Noire. Par rapport aux deux autres régions urbaines du périmètre étudié, il est possible d'affirmer que dans celle de Bâle de nombreuses aires forestières sont également présentes aux alentours directs des aires à empreinte urbaine : $24 \%$ des espaces libres proches des aires de peuplement sont des forêts. Cette situation s'explique par le fait que l'occupation du sol s'est élargie souvent dans les montagnes moyennes et sur le relief collinaire depuis les fonds de vallée jusqu'aux pentes boisées relativement abruptes. Certains domaines forestiers, particulièrement importants pour la récréation de la population urbaine, ont même été conservés à proximité directe de la ville de Bâle.

Les deux indicateurs révèlent de nouveau des valeurs très défavorables à la région urbaine de Mulhouse : ici à peine $30 \%$ de la superficie totale est couverte de forêts et seulement $14,8 \%$ des espaces libres proches des aires d'habitation comportent des forêts. L'essentiel de la couverture forestière revient à la Forêt domaniale de la Hardt, située entre la ville et le Rhin. La Forêt de la Hardt est séparée la plupart du temps des aires d'habitation par une ceinture agricole ou industrielle, ce qui la rend difficilement accessible par le fleuve. Les villages plus éloignés sont également entourés très fréquemment par des aires à usage agricole.

\section{Conclusion}

L'occupation du sol est la plus développée dans la région urbaine bâloise, ce qui est exprimé dans la statistique par le taux le plus élevé d'aires de peuplement. Parallèlement aux aires résidentielles, les aires vouées à l'industrie, à l'activité manufacturière en général et aux transports occupent la part essentielle du périmètre. La région urbaine de Fribourg enregistre le taux d'espace libre le plus élevé, tandis qu'autour de Mulhouse les espaces libres sont à peine supérieurs à ceux de Bâle.

Dans la région urbaine de Bâle, la structure tentaculaire de l'aire continue d'occupation du sol le long des vallées, entrecoupée par d'importants espaces libres, a en fait des répercussions positives : la plupart des habitants peuvent accéder à pied à l'espace libre. La situation de la région urbaine de Fribourg, dont l'aire de peuplement est relativement petite et compacte, comparée à l'échelle interrégionale dans le cadre de l'exploitation de notre base de données, se présente comme la plus favorisée. Celle de Mulhouse par contre, où l'espace environnant est déjà dans un état fort avancé d'occupation du sol diffuse, est la plus défavorisée. 
36 La présente analyse a montré qu'il n'est pas (encore) possible de parler de "ville intermédiaire » dans l'espace du Rhin supérieur méridional. Les régions urbaines de Bâle, Fribourg et Mulhouse sont jusqu'à nos jours nettement séparées par d'amples espaces libres continus. Le contraste ville/campagne demeure clairement exprimé dans ce modèle composé d'aires d'occupation du sol et d'espaces libres. La suppression de la discontinuité territoriale inhérente aux frontières internationales et l'amélioration progressive des moyens de transport interurbains - c'est déjà partiellement le cas en ce qui concerne le système transfrontalier fondé sur le tramway - préfigurent les structures linéaires le long desquelles des aires de peuplement peuvent se développer de manière continue. Il importe désormais de recourir, à l'échelle régionale, à des mesures d'aménagement à long terme susceptibles de contrer les phénomènes qui peuvent conduire au déploiement de villes intermédiaires. La constitution de réserves d'aires non constructibles est à ce sujet une exigence capitale.

En ce qui concerne les régions urbaines prises séparément, nous observons que les régions bâloise et mulhousienne révèlent déjà maintenant des caractéristiques de ville intermédiaire. Le fait que les diverses communes de ces deux portions de l'espace TriRhena aient autorisé trop généreusement la construction d'aires résidentielles et d'activités, ainsi que l'insuffisante coordination en matière d'aménagement régional ont suscité des zones construites incontrôlables et pauvres en potentialités d'identification. Une chance de valorisation et de revitalisation des espaces libres en vue d'un vécu attractif est néanmoins offerte par la concertation et la coopération intercommunale à l'échelle régionale. Sieverts (1999) propose à cet effet le remplacement d'une vision locale du territoire par une planification intégrée, paysagère et urbaine, à vocation régionale. Cette démarche vise à considérer le " paysage » comme un lien, à conserver et à développer, avec la ville intermédiaire. Le concept d'espace libre helvético-franco-allemand pour le Rhin supérieur, ainsi que le projet « Nature et paysage » de l'agglomération trinationale de Bâle (TAB) fournissent des bases de réflexion orientées dans ce sens. Dans l'avenir, la politique et l'aménagement devraient se consacrer à la mise en pratique de ces objectifs, et cela autant dans les trois régions urbaines que dans la Regio TriRhena considérées comme un espace dans son ensemble. Ce n'est qu'à ce prix que la qualité de vie peut être préservée sur ce territoire où vivent 2,2 millions d'habitants.

\section{BIBLIOGRAPHIE}

BUNDESAMT FÜR STATISTIK, BUNDESAMT FÜR UMWELT, WALD UND LANDSCHAFT (1998). - Die Bodennutzung der Schweiz im europäischen Kontext. Integration der Arealstatistik in CORINE Land Cover. Neuchâtel, 43 p.

Busch H., Preuck R., ReckHARd M. (1992). - Potentielle Ruhegebiete für die Erholung im Wohnumfeld und Betroffenheit der Besucher durch Lär. Überlegungen zur Bekämpfung des Lärms in den innerstädischen Grünanalagen. Frankfurt, 38 p. 
DeuTSCHE GESELLSCHAFT FÜR FREIZEIT (1999). — Freizeit in Deutschland. Freizeittrends 2000 plus. Erkrath, Deutsche Gesellschaft für Freizeit, 192 p.

JESSEL B. (1998). - Das Landschaftsbild erfassen und darstellen. Vorschläge für ein pragmatisches Vorgehen, Naturschutz und Landespflege, 30 (11), pp. 356-361.

KAPLAN S. (1988). - « Perception and landscape : conceptions and misconceptions », in NASAR J.L. (sous la direction de) : Environmental aesthetics. Theory, research and applications. Cambridge, pp. 45-55.

KIEMSTEDT H., SCHARPF H. (1990). - « Erholungsvorsorge im rahmen der Lanschaftsplanung”, dans Deutscher rat für Landespflege : Freizeit und erholung - Herausforderungen und Antworten der Landespflege, Schriftenreihe des Deutschen Rates für Landespflege, 57, Meckenheim, pp. 660-663.

MeYRAT-SCHLEE E. (1992). — Mobil sind die Andern - Wohnqualität, Quartierleben und Sesshaftigkeit, Nationales Forschungsprogramm Stadt und Verkehr, 18, Zurich, 157 p.

MÜLLeR H., KRAMER B., FerRANTE C.L. (1997). - Schweizer und ihre Freizeit - Facts und Figures aus 10 Jahren Freizeitforschung, Berner Studien zu Freizeit und Tourismus, 35, Berne, FIF-Université de Berne, $139 \mathrm{p}$.

OpASCHOWSKI H.W. (1997). - Einführung in die Freizeitwissenschaft, Freizeit- und Tourismusstudien, 2, Opladen, Ed. Leske + Budrich, 3. édition augmentée, 333 p.

PRECHTEL N. (1997). - « CORINE - Bodenbedeckungsdaten für Ostdeutschland aus Anwendersicht », Zeitschrift für Photogrammetrie und Fernerkundung, 65 (3), pp. 92-104.

SIEDENTOP S. (1998). - Freiräume in Stadtlandschaften - Eine vergleichende Analyse von Siedlungs- und Freiraumstrukturen ost und westdeutscher Stadtregionen, Rapport de recherche (non diffusé), Institut für ökologische Raumentwicklung, Dresde, $92 \mathrm{p}$.

SIEDENTOP S. (1999). - « Kumulative Landschaftsbelastungen durch Verstädterung - Methodik und Ergebnisse einer vergleichenden Bestandsaufnahme in sechs deutschen Grossstadtregionen ", Natur und Landschaft, 74 (4), pp. 146-155.

SIEVERTS T. (1999). - Zwischenstadt, Bauwelt Fundamente, 118, Brunswick, Wiesbaden, Ed. Vieweg, $191 \mathrm{p}$.

\section{RÉSUMÉS}

Les espaces libres jouent un rôle déterminant sur la qualité des territoires urbains. La présente contribution analyse, au moyen d'une méthode appuyée sur le SIG, la structure des espaces libres et disponibles en faveur des habitants des régions urbaines de Bâle, Fribourg-en-Brisgau et Mulhouse. Les bases statistiques sont constituées par les données sur l'occupation du sol livrées par le système européen harmonisé CORINE. Elles ont été complétées par des statistiques publiques disponibles en Suisse. L'objectif consiste à déterminer des points de repère susceptibles de donner un éclairage sur le développement de la «ville intermédiaire " et de souligner l'importance des espaces libres pour les loisirs de la population résidant en agglomération. Il apparaît que l'urbanisation et la répartition diffuse du peuplement dans les régions urbaines de Bâle et de Mulhouse sont déjà fort avancées. Ici cependant, comme dans la région de Fribourg-enBrisgau, des possibilités subsistent en faveur de la sauvegarde et de l'amélioration de la situation en matière d'espaces libres. 
Land use for recreational purposes is of prime importance in the quality of urban life. Utilizing GIS-methods, this paper analyses the structure and accessibility of open space for recreational uses for the population of the Basle, Friburg and Mulhouse urban areas in the Southern Rhine Valley. The data base is derived from both the European CORINE-land use (satellite) data and the Swiss areal statistics. The objective is to identify indicators for the degree to which urban sprawl ("the city between cities» or "Zwischenstadt») is evident. The results show that urban development and the elimination of open space has progressed immensely in the Basle, Friburg and Mulhouse areas. They also indicate however, that there remain significant potentials and prospects for open space preservation.

Freiflächen spielen in der Diskussion um die Qualität von städtischen Gebieten eine entscheidende Rolle. Dieser Beitrag analysiert mittels einer GIS-gestützten Methodik die Freiflächenstruktur und -verfügbarkeit für die Bewohner der Stadtregionen Basel, Freiburg i. Br. und Mulhouse am südlichen Oberrhein. Grundlage sind die einheitlichen europäischen CORINEBodenbedeckungensdaten, ergänzt um Daten der schweizerischen Arealstatistik. Ziel ist es, Anhaltspunkte über den Grad der entwicklung zur „Zwischenstadt« zu finden und die Bedeutung der Freiräume für die Erholung der Agglomerationsbevölkerung zu unterstreichen. Es zeigt sich, dass die Verstädterung und Zersiedlung in den Stadtregionen Basel und Mulhouse bereits weit fortgeschritten ist, hier aber wie in der Region Freiburg nach wie vor Chancen für eine Sicherung und Verbesserung der Freiraumsituation bestehen.

\section{INDEX}

Schlüsselwörter : Verfügbarkeit von Freiräumen, wohnungsnahe Erholung, Zwischenstadt Mots-clés : détente de proximité, disponibilité d'espaces libres, ville intermédiaire Keywords : accessibility, preservation of open space, recreation, Urban sprawl

\section{AUTEUR}

\section{MARTIN SANDTNER}

Geographisches Institut der Universität Basel - Abteilung Humangeographie - Klingelbergstrasse 16 CH-4056 BASEL 